\title{
Qualidade de voz no estilo de canto heavy metal
}

\section{Voice quality in heavy metal singing}

\author{
Alexsandro Rodrigues Meireles ${ }^{1}$ \\ Frederico Grama Cavalcante ${ }^{2}$ \\ ${ }^{1}$ Universidade Federal do Espírito Santo, Vitória, Espírito Santo, Brasil. \\ meirelesalex@gmail.com \\ 2Universidade Federal do Espírito Santo, Vitória, Espírito Santo, Brasil. \\ fred_cavalcante@hotmail.com
}

\section{Resumo:}

Este artigo analisa a voz cantada em registros altos no estilo heavy metal com a utilização de um perfil de voz baseado na percepção (VPAS). Seu principal objetivo é fornecer um embasamento científico sobre um tema tratado subjetivamente pela literatura musical. Foram utilizados dois cantores profissionais e dois amadores na análise. Suas produções vocais foram analisadas individualmente e, posteriormente, comparadas. Juízes treinados, incluindo professores de canto, fonoaudiólogos e foneticistas, avaliaram essas amostras por meio do perfil de análise de voz VPAS. Os resultados indicam estratégias articulatórias distintas na produção de notas em registros altos nos dois grupos de cantores.

Palavras-chave: voz cantada no estilo heavy metal; protocolo VPAS; qualidade da voz cantada; análise perceptiva do canto.

\section{Abstract:}

This paper studies high registers of heavy metal singing based on the voice profile analysis scheme (VPAS) protocol. Its main objective is to provide a scientific basis for a theme which is subjectively treated by the musical literature. Two professional and two amateur singers were recorded for the perceptual analysis. Their vocal productions were 
analyzed intra- and inter-singers by VPAS experienced judges (vocal coaches, speech therapists, phoneticians). Results indicate that the two groups of singers use distinctive articulatory strategies in singing at high registers.

Keywords: singing in heavy metal; VPAS protocol; voice quality in singing; perceptual analysis of singing.

Data de recebimento: 03/11/2014

Data de aprovação final: 20/09/2015

\section{1 - Introdução}

O heavy metal surgiu como um estilo do rock no início da década de 1970 pela necessidade de se fazer uma música que refletisse a rebeldia de uma geração que, no pósSegunda Guerra Mundial, buscava protestar e transgredir os valores da época por meio de atitudes, vestimentas e do uso do corpo e da voz (BAYER, 2009). Ao se transmitir essa rebeldia pelo canto, criou-se uma técnica vocal mais agressiva, intencionalmente tensa em alguns momentos, e marcada, em alguns estilos, por extremos vocais com notas altíssimas, grande extensão vocal e variação de timbre. Para exemplos desses extremos citamos músicas de bandas de heavy metal clássico, como Iron Maiden e Judas Priest, e de hard rock, como Guns 'N'Roses e Aerosmith. Esses extremos vocais requerem atenção especial quanto à análise clínica da voz, pois podem ser geradores de futuros problemas fonoaudiológicos.

Tecnicamente, cantores desse estilo de música utilizam técnicas vocais variadas rotuladas com o nome de drives vocais, que podem, fisiologicamente, ser produzidos com diferentes configurações do trato vocal, cf. mostrado por PECORARO et al. (2010). Dentre essas técnicas, o belting, utilizado, por exemplo, por Bruce Dickinson do Iron Maiden, é um dos mais utilizados nas regiões mais agudas da voz do hard rock e heavy metal. 
O belting surgiu no teatro musical e expandiu-se para músicas populares diversas como pop music, rhythm and blues, country, rock, heavy metal e jazz. POPEIL (2007, p.79) afirma que as principais características dessa técnica vocal são: a) pregais vogais engrossadas nas bordas; b) músculo vocalis bastante tensionado; c) mudança no padrão de abertura/fechamento das pregas vocais; d) fechamento cíclico rápido das pregas vocais; e) longo grau de fechamento das pregas vocais (acima de 50\%); f) sensação de um aumento do prender a respiração; g) aumento na atividade da mandíbula e nos músculos laríngeos extrínsecos; h) possível movimento anterior do osso hioide; i) posição mais alta da laringe; j) posicionamento mais horizontal da epiglote; k) aumento da pressão subglotal; 1) distribuição regular dos harmônicos amplificados até $4.000 \mathrm{~Hz}$ e energia espectral acima de $15.000 \mathrm{~Hz}$.

O estudo de McCOY (2007) desmistifica alguns preconceitos sobre o belting, a saber: 1) qualidade de voz com harmônicos reforçados nos graves e progressiva diminuição da intensidade dos harmônicos com o aumento da frequência: contrariamente ao esperado, os cantores estudados produziram escalas musicais com reforço de harmônicos desde as notas mais graves até às mais agudas, ou seja, o timbre foi uniforme em toda a extensão vocal; 2) voz extremamente alta e gritada: opostamente à hipótese inicial, os cantores cantaram todas as notas com baixa intensidade e pouco excesso vocal; 3) sinais físicos de desgaste vocal: apesar de cantores líricos frequentemente comentarem esse fato, nenhum desgaste, como mandíbula cerrada, músculos tensos no pescoço e laringes elevadas, foi encontrado. A conclusão principal do trabalho é que a técnica de belting não é prejudicial à saúde vocal, mas sim o mau uso da mesma por cantores amadores, da mesma forma que uma técnica lírica de canto também o é.

GONSALVES et al. (2010, p.196) afirmam haver muitos estudos sobre voz cantada. No entanto, a maioria desses trabalhos estuda cantores de formação erudita, os quais são treinados por métodos de canto tradicionais e constituem minoria entre os cantores. Outros estilos de canto como pop, rock, samba, sertanejo, entre outros, carecem de mais estudos, pois cada estilo musical tem características próprias e técnicas vocais específicas. Os resultados apresentados pelas autoras nesse estudo, baseados em análises perceptivoauditivas, são, a nosso ver contraditórios. Apesar de mostrarem que o rock, dos três estilos analisados, foi o que apresentou maior tensão vocal, esta foi apresentada como leve e 
moderada. Além disso, afirma-se que "tensões musculares não parecem ser afetadas pela técnica vocal e emissões cantadas tensas podem apresentar laringoscopia normal" (GONSALVES et al. 2010, p.199).

Mesmo sendo escassos trabalhos com voz no rock, há alguns estudos na área, como o das autoras supra citadas e as pesquisas de OLIVEIRA e BEHLAU (2004) e THALEN e SUNDBERG (2001). Todavia, o rock destes artigos não é vocalmente relacionado ao estilo aqui estudado. O heavy metal, apesar de ser originário do rock dos anos 1960, cf. declarado por membros de bandas que deram origem ao heavy metal, como Black Sabbath e Motörhead, é uma variação sonora muito mais agressiva do rock tradicional, e ainda menos explorada no meio acadêmico.

Um dos problemas encontrados quando se fala em técnica vocal, apontado por BUTTE et al. (2009), é o fato de a voz cantada ser geralmente descrita subjetivamente pelos professores de canto. Assim, os diferentes estilos de canto são ensinados com base em mudanças fonoarticulatórias subjetivamente experienciadas por esses profissionais. É nesse contexto que surgem expressões como "voz de cabeça", "voz de peito", "voz branca", as quais não possuem fundamento empírico e podem variar significantemente mesmo entre especialistas da voz. Pesquisas anteriores identificaram essa inconsistência e tentaram caracterizar a voz em diferentes estilos através de medidas qualitativas e quantitativas (vide BEZERRA et al., 2009; BUTTE et al., 2009; CLEVELAND, 2001; CURCIO et al., 2000).

Da mesma forma que no estudo de GONSALVES et al. (2010), BUTTE et al. (2009) faz uma análise de diferentes estilos musicais, porém não a direciona ao gênero rock, justificando-se pelo fato de não terem obtido amostras sem "ruído" a níveis aceitáveis para análise, uma vez que, no material por eles analisado, o instrumental já estava contido no áudio da amostra vocal. Por outro lado, diferentemente daquela pesquisa, utilizaramse medidas acústicas para análise dos dados.

O uso da análise acústica se justifica por ser um método não invasivo capaz de prover informações quantitativas sobre os parâmetros fonoarticulatórios do trato vocal. Além disso, a implementação da análise acústica no processamento de sinais de voz permite 
extrair parâmetros de medidas acústicas, dentre os quais, segundo BEHLAU (2001), os mais robustos são a frequência fundamental e seus índices de perturbação, as medidas de ruído e a espectrografia acústica.

Embora a análise acústica seja objetiva e confiável, deve-se aliar a ela a avaliação perceptivo-auditiva da voz (KENT et al, 1999), que, por sua vez, traça um panorama de aspectos vocais tomados pela análise do efeito que o som exerce sobre o ouvinte. Com esse intuito, o grupo de pesquisa liderado por John Laver na década de 1980, quando a análise acústica por meio digital ainda era embrionária, desenvolveu um perfil de descrição da qualidade vocal baseado na percepção de traços auditivos, acústicos, articulatórios e fisiológicos (Voice Profile Analysis Scheme, VPAS, cf. LAVER et al., 1981), o qual deu embasamento a estudos posteriores da avaliação perceptivo-auditiva da voz.

CAMARGO e MADUREIRA (2008a) traduziram o VPAS e adaptaram-no ao português brasileiro, criando um protocolo de qualidade de voz de referência na análise fonética e fonoaudiológica do Brasil. Este perfil, porém, não é específico para a voz cantada, sendo necessária uma adaptação do modelo para esse fim. Dentre os aspectos não mencionados nesse método, por exemplo, estão os ajustes epiglotais e de falsas pregas vocais, os quais são empregados em certos tipos de drives vocais como os usados no singing throat da Mongólia e na voz gutural no estilo death metal (ex. Max Cavalera da banda Sepultura).

Além dos aspectos mencionados anteriormente sobre o estudo do canto popular, outro fator pouco avaliado na relação da fonética acústica com a avaliação perceptual é o vibrato. No entanto, vibrato é um dos ornamentos frequentemente utilizados em diversos estilos de canto e essencial para uma boa técnica vocal. SATALOFF (1991) e BEZERRA et al. (1991) concluíram que o vibrato pode ser produzido de diferentes maneiras, para cada gênero da voz cantada. Em um outro estudo, CURCIO et al. (2000) estudaram o vibrato em três diferentes estilos musicais: ópera, rock e sertanejo, utilizando amostras extraídas de cds de aúdio, disponíveis comercialmente. Essas análises, porém, não abrangeram outros estilos, que possuem suas particularidades técnicas. 
Pelos motivos aqui apresentados, pela falta de material didático direcionado ao estilo heavy metal, cuja popularidade e difusão são grandes, e pela necessidade de técnicas e pedagogia vocais aliadas ao amparo científico, justifica-se uma análise da voz cantada, sob a ótica da análise perceptivo-auditiva do VPAS e das análises acústicas. Além disso, existem poucas pesquisas publicadas que correlacionam os dados perceptivo-auditivos à análise acústica da voz cantada, tema este que será abordado em nossos trabalhos futuros.

\section{2 - O protocolo VPAS}

LAVER (1980), com base no trabalho de ABERCROMBIE (1967), define qualidade de voz como todas as características vocais relacionadas à fala de um indivíduo, incluindo aspectos laríngeos, supralaríngeos e de dinâmica vocal. Assim são estudados tanto aspectos linguísticos quanto extralinguísticos e paralinguísticos da fala. É importante ressaltar que na análise da qualidade de voz de um falante devemos considerar apenas os aspectos vocais que ocorrem frequentemente na fala de um indivíduo. Segundo LAVER (1994, p.152), essas características de longo termo na voz correspondem a "todos os fatores que podem ser potencialmente prolongados além do domínio do segmento" (tradução nossa).

A partir dos estudos de Laver, um grupo de pesquisadores do Queen Margareth University College na Escócia desenvolveu o protocolo de análise de qualidade vocal VPAS (voice profile analysis scheme, vide LAVER et al., 1981; LAVER, 2000; MACKENZIE-BECK, 2005; LAVER e MACKENZIE-BECK, 2007). A base do protocolo reside na configuração neutra do trato vocal, semelhantemente à posição articulatória para a vogal schwa. A partir desse ajuste neutro, descrevem-se os fatores desviantes dessa configuração vocal. LAVER (1980) menciona 53 tipos possíveis de ajuste.

Conforme salientam CAMARGO e MADUREIRA (2008a), "the adoption of the neutral setting as reference can be considered a landmark in the investigation of voice qualities 
since it does not introduce a rupture between normality and voice disorder". Além disso, essa metodologia i) permite a comparação de qualidade vocal entre dialetos e até línguas diferentes, pois o padrão neutro de configuração do trato vocal é universal; e ii) elimina a clássica dicotomia entre fala normal e fala patológica. Nesse protocolo, qualquer tipo de ajuste de qualidade vocal é possível de ser reproduzido, o que impede de, a priori, afirmarmos se um determinado tipo de qualidade de voz é proveniente de uma patologia na fala.

O ajuste neutro do trato consiste basicamente de: i) pregas vocais com vozeamento pleno sem escape de ar audível; ii) trato vocal sem expansão ou constrição saliente; iii) tamanho do trato sem alongamento ou encurtamento; iv) tensão moderada no trato vocal e nas pregas vocais; v) véu palatino levantado; e vi) mandíbula ligeiramente aberta. Ressaltamos que os desvios desse ajuste neutro só são considerados a longo termo. Sendo assim, só podemos considerar um determinado ajuste de qualidade vocal se não for característica articulatória inerente de um segmento enunciado. Ex: no português brasileiro, toda vogal posterior é arredondada. Assim, não poderíamos mencionar um determinado grau de ajuste labial nesse caso. Por outro lado, se tivermos arredondamento em vogais altas, no português, consideramos um ajuste labial, pois, nesse caso, arredondamento não é inerente a estes tipos de segmentos.

Como podemos ver na tabela da Figura 1, o protocolo VPAS de 2007 (adaptado para o português brasileiro por CAMARGO e MADUREIRA, 2008a) é dividido em 3 grandes blocos: 1) elementos do trato vocal (ajustes supralaríngeos); 2) tensão muscular geral (ajustes laríngeos e supralaríngeos); 3) elementos fonatórios (ajustes laríngeos). Além desses ajustes, são descritos 4 parâmetros de dinâmica vocal: 1) pitch (variação de frequência fundamental (f0)); 2) intensidade (variação em dB); 3) tempo (inclui-se aqui a continuidade de fala e a taxa de elocução); e 4) elementos de suporte respiratório.

De acordo com LAVER e MACKENZIE-BECK (2007), vários princípios estão presentes no modelo de qualidade vocal, a saber: i) interdependência; ii) compatibilidade; e iii) susceptibilidade. A interdependência relaciona-se à interação fisiológica entre ajustes distintos. Ex: um ajuste ponta de língua avançada é concomitante a um ajuste de corpo de língua avançado. A compatibilidade relaciona-se à natureza conflitante de determinados 
ajustes. Ex: um ajuste ponta de língua recuada é conflitante com um ajuste ponta de língua avançada. A susceptibilidade refere-se à vulnerabilidade de um segmento a um ajuste. Grosso modo, quanto mais distinto articulatoriamente o ajuste do segmento-alvo, mais vulnerável é o segmento. Ex: as vogais não-arredondadas são mais susceptíveis a um ajuste de arredondamento de lábios.

\begin{tabular}{|c|c|c|c|c|c|c|c|c|c|c|}
\hline \multirow[t]{3}{*}{ QUALIDADE VOCAL } & \multicolumn{2}{|c|}{ PRIMEIRA PASSADA } & \multicolumn{8}{|c|}{ SEGUNDA PASSADA } \\
\hline & \multirow{2}{*}{ Neutro } & \multirow{2}{*}{$\begin{array}{l}\text { Não } \\
\text { neutro }\end{array}$} & \multirow{2}{*}{\multicolumn{2}{|c|}{ AJUSTE }} & \multicolumn{3}{|c|}{ Moderado } & \multicolumn{3}{|c|}{ Extremo } \\
\hline & & & & & 1 & 2 & 3 & 4 & 5 & 6 \\
\hline \multicolumn{11}{|c|}{ A. ELEMENTOS DO TRATO VOCAL } \\
\hline \multirow{5}{*}{ 1.Lábios } & & & \multicolumn{2}{|c|}{ Arredondados/protraídos } & & & & & & \\
\hline & & & \multicolumn{2}{|c|}{ Estirados } & & & & & & \\
\hline & & & \multicolumn{2}{|c|}{ Labiodentalização } & & & & & & \\
\hline & & & \multicolumn{2}{|c|}{ Extensão diminuída } & & & & & & \\
\hline & & & \multicolumn{2}{|c|}{ Extensão aumentada } & & & & & & \\
\hline \multirow{5}{*}{ 2. Mandíbula } & & & \multicolumn{2}{|c|}{ Fechada } & & & & & & \\
\hline & & & \multicolumn{2}{|l|}{ Aberta } & & & & & & \\
\hline & & & \multicolumn{2}{|l|}{ Protraída } & & & & & & \\
\hline & & & \multicolumn{2}{|c|}{ Extensão diminuída } & & & & & & \\
\hline & & & \multicolumn{2}{|c|}{ Extensão aumentada } & & & & & & \\
\hline 3.Língua ponta/lâmina & & & Avançad & & & & & & & \\
\hline & & & Recuada & & & & & & & \\
\hline 4. Corpo de língua & & & Avançad & & & & & & & \\
\hline & & & Recuado & & & & & & & \\
\hline & & & Elevado & & & & & & & \\
\hline & & & Abaixado & & & & & & & \\
\hline & & & Extensão & ninuída & & & & & & \\
\hline & & & Extensão & mentada & & & & & & \\
\hline 5.Faringe & & & Constriçã & & & & & & & \\
\hline & & & Expansã & & & & & & & \\
\hline 6.Velofaringe & & & Escape $n$ & audível & & & & & & \\
\hline & & & Nasal & & & & & & & \\
\hline & & & Denasal & & & & & & & \\
\hline 7. Altura de laringe & & & Elevada & & & & & & & \\
\hline & & & Abaixada & & & & & & & \\
\hline B. TENSÃO MUSCULAR & RAL & & & & & & & & & \\
\hline 8. Tensão do trato vocal & & & Hiperfun & & & & & & & \\
\hline & & & Hipofunç & & & & & & & \\
\hline 9. Tensão laríngea & & & Hiperfun & & & & & & & \\
\hline & & & Hipofunç & & & & & & & \\
\hline C. ELEMENTOS FONATO & OS & & & & & & & & & \\
\hline & AJUSTE & & Presente & & & us & le es: & cal & & \\
\hline & & & Neutro & & & der & do & & rem & \\
\hline & & & & Neutro & 1 & 2 & 3 & 4 & 5 & 6 \\
\hline 10. Modo de fonação & Modal & & & & & & & & & \\
\hline & Falsete & & & & & & & & & \\
\hline & Crepitânc & vocal fry & & & & & & & & \\
\hline & Voz crepi & & & & & & & & & \\
\hline 11. Fricção laríngea & Escape d & & & & & & & & & \\
\hline & Voz sopro & & & & & & & & & \\
\hline 12.Irregularidade laríngea & Voz áspe & & & & & & & & & \\
\hline
\end{tabular}

Figura 1: Figura 1a: Protocolo VPAS para análise dos ajustes da qualidade de voz. Para maiores detalhes vide texto. 


\begin{tabular}{|c|c|c|c|c|c|c|c|c|c|}
\hline DINÂMICA VOCAL & & Neutro & AJUSTE & \multicolumn{3}{|c|}{ Moderado } & \multicolumn{3}{|c|}{ Extremo } \\
\hline \multicolumn{10}{|c|}{ D. ELEMENTOS PROSÓDICOS } \\
\hline \multirow{6}{*}{ 13.Pitch (fo) } & \multirow[t]{2}{*}{ Habitual } & & Elevado & & & & & & \\
\hline & & & Abaixado & & & & & & \\
\hline & \multirow[t]{2}{*}{ Extensão } & & Diminuída & & & & & & \\
\hline & & & Aumentada & & & & & & \\
\hline & \multirow[t]{2}{*}{ Variabilidade } & & Diminuída & & & & & & \\
\hline & & & Aumentada & & & & & & \\
\hline \multirow{6}{*}{$\begin{array}{l}\text { 14.Loudness } \\
\text { (intensidade) }\end{array}$} & \multirow[t]{2}{*}{ Habitual } & & Aumentado & & & & & & \\
\hline & & & Diminuído & & & & & & \\
\hline & \multirow[t]{2}{*}{ Extensão } & & Diminuída & & & & & & \\
\hline & & & Aumentada & & & & & & \\
\hline & \multirow[t]{2}{*}{ Variabilidade } & & Diminuída & & & & & & \\
\hline & & & Aumentada & & & & & & \\
\hline \multicolumn{10}{|l|}{ 15. Tempo } \\
\hline \multicolumn{3}{|l|}{ Continuidade } & Interrompida & & & & & & \\
\hline \multirow{2}{*}{\multicolumn{2}{|c|}{ Taxa de elocução }} & & Rápida & & & & & & \\
\hline & & & Lenta & & & & & & \\
\hline \multicolumn{10}{|c|}{ 16.OUTROS ELEMENTOS } \\
\hline \multirow{2}{*}{\multicolumn{3}{|c|}{ Suporte respiratório }} & Adequado & & & & & & \\
\hline & & & Inadequado & & & & & & \\
\hline
\end{tabular}

Figura 1: Figura 1b (continuação): Protocolo VPAS para análise dos ajustes da qualidade de voz.

A aplicação do protocolo VPAS é composta por dois passos. No primeiro passo, apenas define-se se há um ajuste não neutro. Caso haja, passa-se ao segundo passo, em que os ajustes são divididos entre moderados (1 a 3) e extremos (4 a 6). CAMARGO e MADUREIRA (2008b, p.80) resumem os ajustes da seguinte forma: 1 - pequena diferença em relação ao ajuste neutro; 2 - leve diferença em relação ao ajuste neutro; 3 moderada diferença em relação ao ajuste neutro; 4 - notável diferença em relação ao ajuste neutro; 5 - marcante diferença em relação ao ajuste neutro; e 6 - extrema diferença em relação ao ajuste neutro. 
CAMARGO e MADUREIRA (2008b, p.95) atestam a validade do VPAS, o qual propicia “o rompimento da linha divisória entre normalidade e alteração vocal, permitindo que este foco de análise seja usado em variadas situações de inclusão do tema voz, tais como reabilitação de alterações, bem como assessoria no sentido de uso profissional da voz."

Ampliando ainda mais as possibilidades de uso para voz profissional do VPAS, propomos uma aplicação inédita, até onde sabemos, deste protocolo: a análise da voz cantada. Dadas as especificidades técnicas deste tema, teremos de fazer, futuramente, alguns ajustes em seus parâmetros para uma melhor descrição da qualidade de voz no canto.

\section{3 - Metodologia}

Quatro cantores de faixa etária entre 20 e 40 anos, sendo 2 experientes com mais de 15 anos de prática de canto (informantes J e A) e 2 amadores com menos de 3 anos de prática de canto (informantes A2 e I)) cantaram integralmente a música Aces High, da banda Iron Maiden, com playback instrumental tocado em fones de ouvido. O playback serviu para auxiliá-los quanto a tom e uniformidade do tempo de gravação. Apenas o áudio da voz dos cantores foi registrado, para que não houvesse interferências da harmonia na avaliação da amostra. A produção do material para análise acima referido foi realizada no estúdio de gravação do Departamento de Comunicação da UFES (LABCOM) para o informante A e no estúdio particular Ocean Studios para os informantes J, A2 e I. Ambas gravações foram feitas com qualidade de gravação profissional com taxa de amostragem de $44,1 \mathrm{kHz}$ estéreo em arquivo .wav. No primeiro estúdio utilizou-se um microfone condensador e no segundo estúdio um microfone cardioide unidirecional. Além do áudio, foi também capturado o vídeo das performances vocais.

Os cantores gravados se adequaram ao perfil esperado, comprovado após a utilização de um questionário informal para avaliar o perfil histórico-musical dos indivíduos selecionados. Os cantores experientes adequam-se ao perfil, pois o Informante A canta há mais de 15 anos e é vocalista numa banda cover do Iron Maiden, e o Informante J 
canta há mais de 15 anos e possui graduação em canto pela Faculdade de Música do Espírito Santo, além de ser vocalista de uma banda de heavy metal famosa no Espírito Santo. Já os informantes inexperientes A2 e I não tem experiência alguma em cantarem em registros tão altos e não cantam em nenhuma banda de heavy metal. O informante I é iniciante no estudo do canto e o informante A2 estudou canto por apenas 4 meses.

Selecionou-se 2 trechos mais agudos da música Aces High (Figuras 2 e 3), a fim de se verificar as estratégias vocais dos cantores experientes para se cantar em registros extremos da extensão da voz. Os trechos selecionados foram as partes 1 e parte 2 nas Figuras 2 e 3 abaixo:

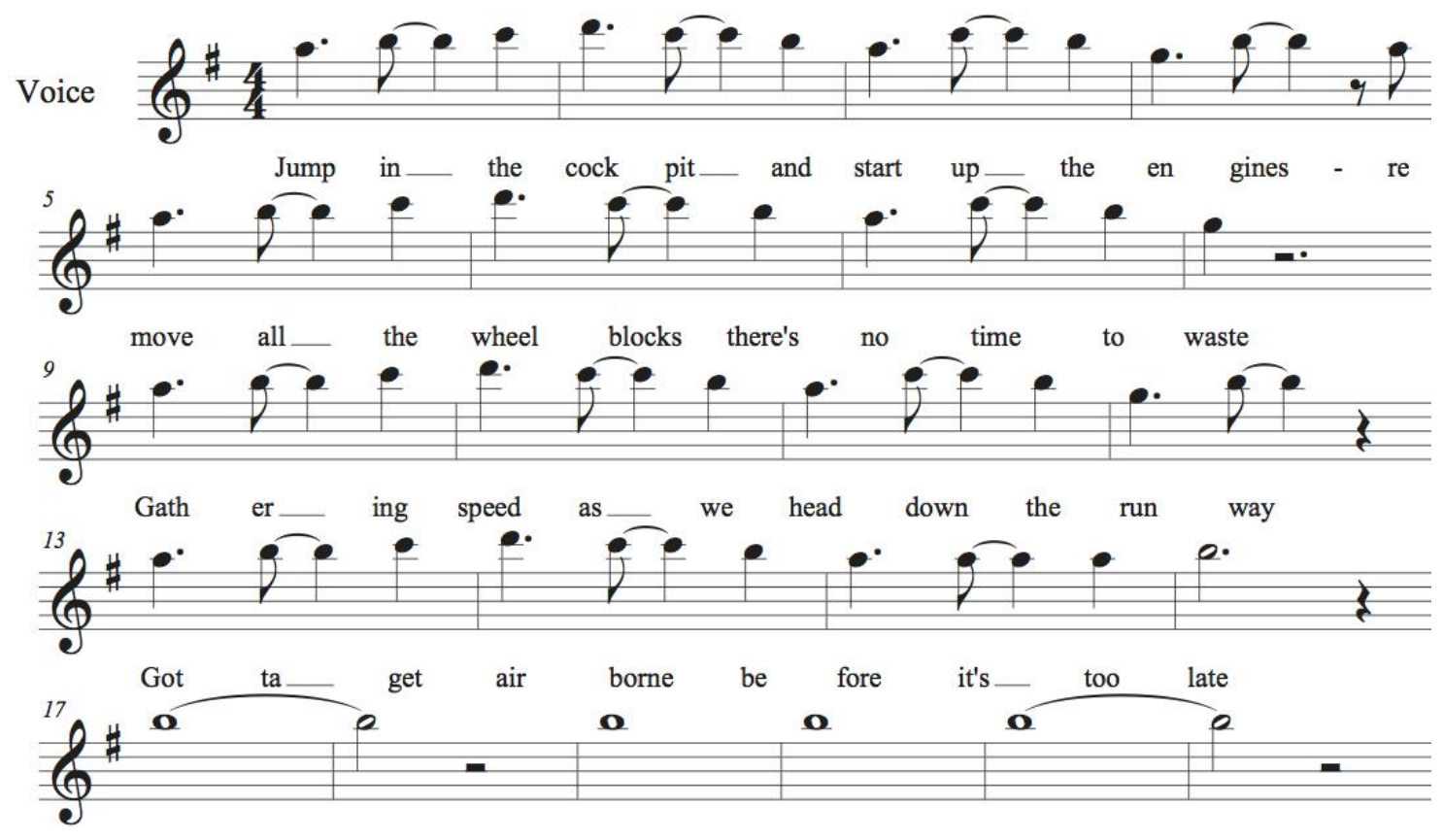

Figura 2: Parte 1 com notas agudas de Aces High analisado pelo VPAS. 


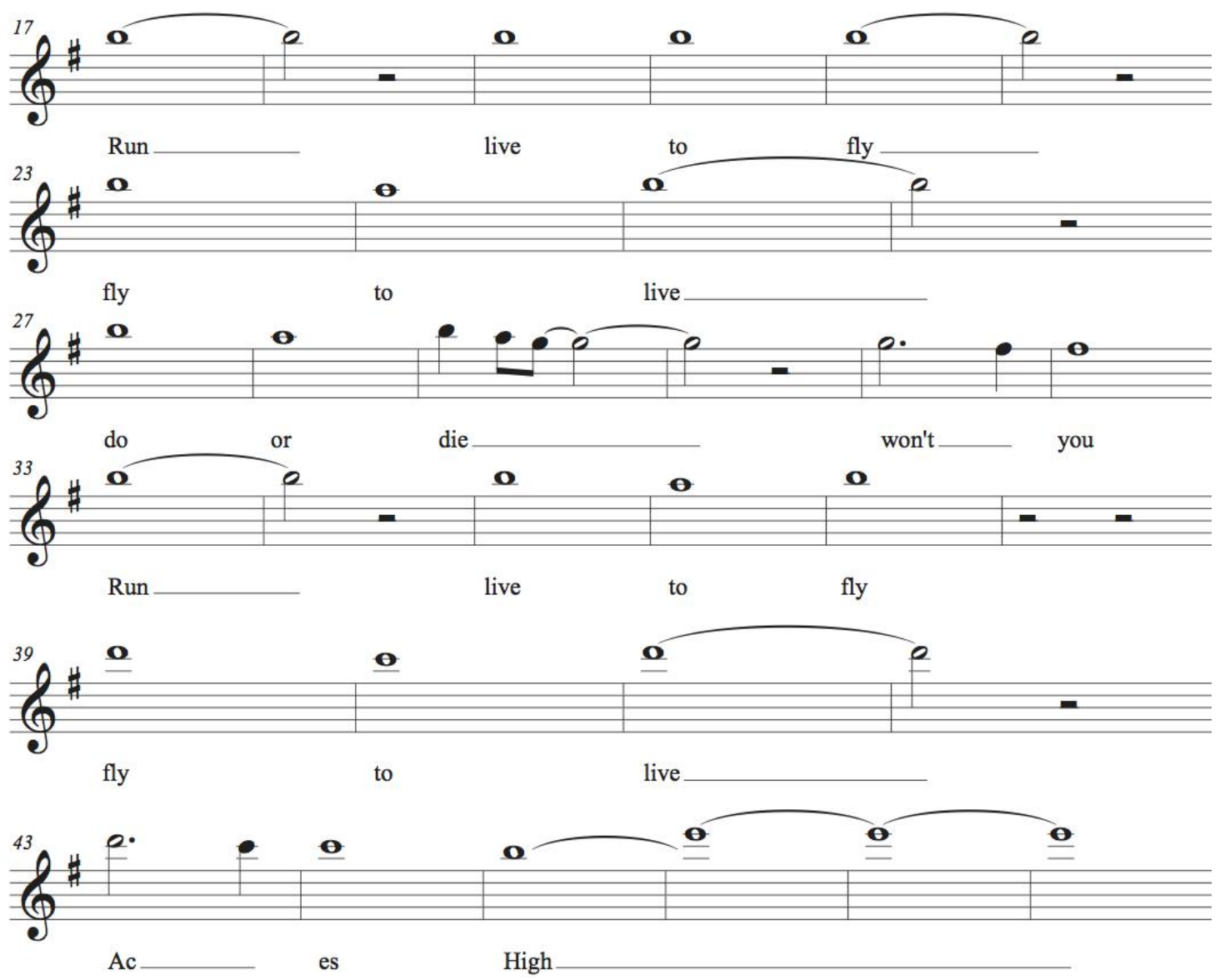

Figura 3: Parte 2 com notas agudas de Aces High analisado pelo VPAS.

Após a preparação do material de amostragem, os dados foram submetidos a uma análise perceptivo-auditiva do canto, gerada a partir dos esquemas desenvolvidos por John Laver e colegas (VPAS, LAVER et al., 1981; LAVER, 2000; MACKENZIE-BECK, 2005; LAVER e MACKENZIE-BECK, 2007) e modificados por CAMARGO e MADUREIRA (Brazilian Portuguese VPAS, CAMARGO e MADUREIRA, 2008a). Os avaliadores, experientes na análise VPAS, foram 3 fonoaudiólogos, 1 foneticista e 1 cantor lírico, professores e/ou estudantes de pós-graduação da PUC-SP, foco de estudos do VPAS no Brasil. 


\section{4 - Resultados}

Os resultados das análises perceptivas utilizando-se o protocolo VPAS estão, devido ao tamanho, relacionados nas figuras 4 a 7 do Anexo 1. Através dessas análises, podemos notar estratégias distintas na produção de notas em registros altos nos dois grupos de cantores.

Os cantores amadores (I, A2) apresentaram menos ajustes vocais para cantar notas altas extremas (acima do dó de peito do tenor). Os juízes, pois, observaram para estes cantores apenas ajustes de tensão muscular geral e ajustes fonatórios. Ambos obtiveram o grau 3 em hiperfunção do trato vocal e hiperfunção da tensão laríngea. No entanto, como esses cantores utilizaram estratégias diferentes para realização das notas musicais, diferentes ajustes fonatórios foram percebidos.

O cantor A2 utilizou voz modal no trecho precedente à parte 1 analisada [00:00 a 00:14] ${ }^{1}$ e falsete durante toda a parte 1 [00:15 a 00:30]. Na parte 2 analisada utilizou voz modal no trecho "Run, live to fly, fly to live, do or die. Run" [00:45 a 01:00] e falsete no trecho "live to fly, fly to live. aces high" [01:01 a 01:13]. Os trechos em que falsete foi utilizado são os correspondentes às notas mais altas da canção. Por sua vez, o cantor I utilizou uma voz não modal áspera com escape de ar, predominantemente, e uma voz soprosa intermitentemente, durante toda a música. Esse tipo de ajuste laríngeo pode ser chamado de drive vocal, porém, devido à baixa intensidade do som, não é o padrão esperado neste estilo de canto.

Os cantores experientes, por outro lado, apesar de também utilizarem ajustes de tensão muscular geral, apresentaram graus mais elevados desses ajustes. Para hiperfunção do trato vocal e hiperfunção da tensão laríngea, o cantor A obteve o valor 5 e o cantor J, o valor 6. Além disso, encontramos os seguintes ajustes, segundo a tabela VPAS (Figura 1): mandíbula aberta (informante A, ajuste 2; informante J, ajuste 4) elevação da laringe

\footnotetext{
${ }^{1} \mathrm{O}$ tempo aqui descrito começa no momento em que o vocalista começa a cantar no playback de Aces High.
} 
(informante $\mathrm{A}$, ajuste 4; informante $\mathrm{J}$, ajuste 5), extensão diminuída da mandíbula (informante $\mathrm{J}$, ajuste 2), corpo de língua recuado (informante $\mathrm{J}$, ajuste 2), extensão diminuída do corpo de língua (informante J, ajuste 2). Quanto aos ajustes fonatórios, predominou em ambos os cantores a voz modal com voz áspera intermitente. Esse tipo de ajuste corresponde ao chamado na literatura de drive vocal do heavy metal.

\section{5 - Discussão e Conclusões}

Levando-se em consideração o fato de que "o protocolo VPAS, [...] foi desenvolvido com base na habilidade perceptiva dos juízes em ouvir as vozes enquanto combinações de ajustes complementares, cujo método pode ser aprendido" (LAVER et al, 1991; LAVER, 2000; MACKENZIE-BECK, 2005; Camargo e Madureira, 2008b, p. 89), constatamos a validade de aplicação do protocolo para os estudos do canto, uma vez que essas "combinações de ajustes complementares" tratam-se de ajustes musculares de longo termo das estruturas do aparelho fonador.

O modelo de LAVER et al. (1991), contudo, é baseado em pistas auditivas acústicas, fisiológicas e articulatórias do aparelho fonador durante a emissão sonora. Assim, a análise da aplicação do protocolo será melhor se for aliada aos resultados da análise acústica dos excertos auditivos. Este procedimento será feito em uma etapa futura desta pesquisa.

Dos protocolos de voz encontrados na literatura, constatamos que o VPAS é o mais completo para uma análise do material de canto. Outros protocolos, como o RASAT (PINHO e PONTES, 2002), adaptação para o português da escala GRBAS, que é uma escala perceptual para avaliação dos parâmetros rouquidão (R), aspereza (A), soprosidade (S), astenia (A) e tensão da voz (T), limitam-se à análise dos aspectos fonatórios e voltamse à análise clínica. Já o protocolo VPAS pode ser aplicado a qualquer tipo de material de voz. 
Com relação ao material de canto analisado, observou-se, apesar da nítida distinção entre estratégias articulatórias de cantores amadores (informantes A2 e I) e experientes (informantes A e J), também estratégias individuais para se cantar em registros extremos. A principal distinção notada entre os cantores estudados foi a manutenção contínua de uma grande tensão no trato vocal e nas pregas vocais pelos cantores experientes, a qual foi encontrada intermitentemente e em menor grau nos cantores amadores. Além disso, os cantores experientes, a fïm de facilitar alcançar as notas altas utilizaram uma postura vocal constante de mandíbula aberta e laringe alta, ajustes ausentes nos cantores amadores.

Em acréscimo, é importante ressaltar a maior dificuldade em se atingir as notas mais altas da música pelo cantor J, em comparação ao cantor A. Segundo J, essa dificuldade não tem relação direta com a altura das notas, mas com a forma de se cantar este estilo vocal agressivo de heavy metal. O sujeito diz, inclusive, ser capaz de cantar notas muito mais altas em sua banda de heavy metal melódico, porém com uma configuração vocal distinta do estilo estudado. Dessa forma, precisamos comparar, futuramente, quais os ajustes realizados no estilo de canto de $\mathrm{J}$ para se alcançar notas ainda mais altas.

\section{6 - Considerações finais}

Posteriormente, os perfis vocais avaliados pelo VPAS serão comparados aos resultados das análises acústicas. Além da análise individual dos dados coletados, as medidas acústicas e o protocolo perceptivo-auditivo serão comparados entre os cantores (amadores $\mathrm{x}$ experientes), a fim de se estabelecer critérios objetivos para o ensino do canto heavy metal em registros altos. Por fim, os resultados da análise serão tratados estatisticamente através de testes paramétricos e não-paramétricos, para se observar se as diferenças acústico-perceptivas entre os cantores são estatisticamente significantes.

Por fim, será imprescindível uma reformulação do protocolo VPAS, visto que muitos ajustes disponibilizados são irrelevantes para o canto e outros ajustes estão ausentes no 
protocolo (como taxa de vibrato e ajustes laríngeos relacionados aos drives vocais). Dessa maneira, pretendemos gravar uma base de dados para definição dos parâmetros ideais para o estudo dos diversos tipos de canto.

\section{7 - Agradecimentos}

Às Dras. Zuleica Camargo e Sandra Madureira, bem como seus alunos do Programa de Pós-Graduação em Linguística Aplicada e Estudos da Linguagem (LAEL) da PUC-SP, pela avaliação perceptiva desta pesquisa. À Fapes e ao CNPq, pelo financiamento da pesquisa. Aos pareceristas, pelos comentários pertinentes.

\section{Referências}

1. ABERCROMBIE, D. (1967). Elements of general phonetics. Edinburgh: Edinburgh University Press.

2. BAYER, G (2009). Heavy metal music in Britain. London: Ashgate popular and folk music series.

3. BEHLAU, M. (Org.). (2001). Voz. O livro do especialista. vol I. 1a ed. Rio de Janeiro: Revinter.

4. BEZERRA, A.; CUKIER-BLAJ, S.; DUPRAT, A.; CAMARGO, Z.; GRANATO, L. (2009). "The Characterization of the Vibrato in Lyric and Sertanejo Singing Styles: Acoustic and Perceptual Auditory Aspects". Journal of Voice. v.23, p.666-670.

5. BUTTE, C; ZHANG, Y; SONG, H.; JIANG, J. (2009). "Perturbation and Nonlinear Dynamic Analysis of Different Singing Styles". Journal of Voice. v.23, p.647-652.

6. CAMARGO, Z.; MADUREIRA, S. "Voice quality analysis from a phonetic perspective: Voice Profile Analysis SchemeProfile for Brazilian Portuguese (BP-VPAS)" (2008a). In: Fourth Conference on Speech Prosody - Abstract book and Proceedings. Campinas : Capes, Fapesp, CNPq. v.1, p.57-60.

7. (2008b) “Avaliação vocal sob a perspectiva fonética: análise preliminar”. Distúrbios da Comunicação. v.20(1), p.77-96.

8. CLEVELAND, T; SUNDBERG, J.; STONE, R. (2001). "Long-term-average spectrum characteristics of country singers during speaking and singing". Journal of Voice. v.15, p.54-60. 
9. CURCIO D.; BEHLAU M.S.; PONTES P.L. (2000). "Estudo da freqüência do vibrato em três estilos de canto". In: Sociedade Brasileira de Fonoaudiologia - VII Encontro Nacional de Fonoaudiologia. Artcolor: São Paulo.

10. KENT, R. D.; WEISMER, G.; KENT, J.F; VORPERIAN, H.K.; DUFFY, J.R. (1999). "Acoustic studies of dysarthric speech: methods, progress, and potential". Journal of Communication Disorders, v.32, p.141-186.

11. GONSALVES, A.; AMIN, E.; BEHLAU, M. (2010). “Análise do grau global e tensão da voz em cantores de roque”. Pró-Fono R. Atual. Cient. [online]. vol.22, n.3, p.195200.

12. LAVER, J. (1980). The phonetic description of voice quality. Cambridge: Cambridge: Cambridge University Press.

13. LAVER, John. (1994). Principles of phonetics.Cambridge: Cambridge University Press.

14. LAVER, J. (2000). "Phonetic evaluation of voice quality". In: Voice quality measurement”. R.D Kent, Ball M.J. (ed). San Diego: Singular Publishing, p.37-48.

15. LAVER, J; WIRS, S; MACKENZIE, J; HILLER, S.M. (1981). A perceptual protocol for the analysis of vocal profiles. Edinburgh; Edinburg University.Department of Linguistics; p.139-55. [Workin Progress, 14].

16. LAVER, J; MACKENZIE-BECK, J. (2007). Vocal Profile Analysis Scheme VPAS. Queen Margareth University College-QMUC, Speech Science Research Centre, Edinburgh.

17. MACKENZIE-BECK, J. (2005). "Perceptual analysis of voice quality: the place of vocal profile analysis". In: A figure of speech: a festschrift for John Laver. W.J. Hardcastle, J. Mackenzie-Beck (ed). Mahwah: Lawrence Erlbrum, p.285-322.

18. McCOY, S. (2007). “A classical pedagogue explores belting”. Journal of Singing, vol.63, p.545-49.

19. OLIVEIRA, L; BEHLAU, M. (2004) Perfil vocal de cantores amadores de banda de roque. [monografia]. São Paulo(SP): Centro de Estudos da Voz.

20. PECORARO, G; DUPRAT, A.; BANNWARTH, S.; ANDRADA e SILVA, M. (2010). "Cantores de rock: ajustes dinâmicos de trato vocal, análise perceptivoauditiva e acústica das vozes ao longo de cinco décadas”. In: Anais do $18^{\circ}$ Congresso Brasileiro de Fonoaudiologia. Curitiba.

21. PINHO, S.; PONTES, P. (2002). Escala de avaliação perceptiva da fonte glótica: RASAT. Vox Brasilis. v.8(3).

22. POPEIL, L. S. (2007). "The multiplicity of belting”. Journal of Singing, v.64, n.1, p.77-80.

23. SATAlOFF, R. T. (1991). Professional Voice: The Science and Art of Clinical 
Care. New York: Raven Press.

24. THALEN, M; SUNDBERG, J. (2001). "Describing different styles of singing- a comparison of a female singer's voice source in "Classical", "Pop", "Jazz", and “'Blues”. Log Phon Vocol. v.26, p.82-93, 2001.

Notas sobre os autores

Alexsandro Rodrigues Meireles é Professor Adjunto IV na Universidade Federal do Espírito Santo lecionando disciplinas e realizando pesquisa na área de Linguística e Música, Bacharelando em Música pela UFES (Habilitação: Composição com Ênfase em Trilha Musical), Mestre em Letras: Estudos Linguísticos pela UFMG (2001) e Doutor em Linguística pela UNICAMP. Tem experiência na área de Linguística/Música, com ênfase em Fonética e Fonologia, atuando principalmente nos seguintes temas: taxa de elocução, ritmo de fala, sistemas dinâmicos, fonética acústica e articulatória, fonologia experimental, qualidade de voz. No Programa de Pós-Graduação em Linguística da UFES leciona disciplinas de fonética/fonologia e coordena o Laboratório de Fonética vinculado ao Núcleo de Estudos em Fonética e Fonologia (NEFF). Atualmente é membro do Conselho de Curadores da UFES.

Frederico Grama Cavalcante é Graduado em Música: Licenciatura na Universidade Federal do Espírito Santo e aluno do Curso de Formação Musical em Canto Lírico, da Faculdade de Música do Espírito Santo (FAMES). Possui as seguintes pesquisas concluídas: "Estudo fonético-acústico da variação rítmica no dialeto mineiro" (2011), "Análise acústica e perceptivo-auditiva do canto no heavy metal" (2012) e "Análise acústico-perceptiva dos vocalizes no canto lírico (2013)”. Atualmente é preparador vocal do Madrigal Mosaico do Curso de Música da UFES. 


\section{Anexos}

\begin{tabular}{|c|c|c|c|c|c|c|c|c|c|c|}
\hline \multirow{3}{*}{\begin{tabular}{|l|} 
QUALIDADE VOCAL \\
\\
\end{tabular}} & \multicolumn{2}{|c|}{ PRIMEIRA PASSADA } & \multicolumn{8}{|c|}{ SEGUNDA PASSADA } \\
\hline & \multirow[t]{2}{*}{ Neutro } & \multirow{2}{*}{\begin{tabular}{|l|} 
Não \\
neutro
\end{tabular}} & \multirow{2}{*}{\multicolumn{2}{|c|}{ AJUSTE }} & \multicolumn{3}{|c|}{ Moderado } & \multicolumn{3}{|c|}{ Extremo } \\
\hline & & & & & 1 & 2 & 3 & 4 & 5 & 6 \\
\hline \multicolumn{11}{|c|}{ A. ELEMENTOS DO TRATO VOCAL } \\
\hline \multirow{5}{*}{ 1.Lábios } & & & \multicolumn{2}{|c|}{ Arredondados/protraídos } & & & & & & \\
\hline & & & \multicolumn{2}{|c|}{ Estirados } & & & & & & \\
\hline & & & \multicolumn{2}{|c|}{ Labiodentalização } & & & & & & \\
\hline & & & \multicolumn{2}{|c|}{ Extensão diminuída } & & & & & & \\
\hline & & & \multicolumn{2}{|c|}{ Extensão aumentada } & & & & & & \\
\hline \multirow{5}{*}{ 2. Mandíbula } & & & \multicolumn{2}{|c|}{ Fechada } & & & & & & \\
\hline & & & \multicolumn{2}{|l|}{ Aberta } & & & & & & \\
\hline & & & \multicolumn{2}{|c|}{ Protraída } & & & & & & \\
\hline & & & \multicolumn{2}{|c|}{ Extensão diminuída } & & & & & & \\
\hline & & & \multicolumn{2}{|c|}{ Extensão aumentada } & & & & & & \\
\hline 3.Língua ponta/lâmina & & & Avançac & & & & & & & \\
\hline & & & Recuad & & & & & & & \\
\hline 4. Corpo de língua & & & Avançac & & & & & & & \\
\hline & & & Recuad & & & & & & & \\
\hline & & & Elevado & & & & & & & \\
\hline & & & Abaixad & & & & & & & \\
\hline & & & Extensã & minuída & & & & & & \\
\hline & & & Extensã & umentada & & & & & & \\
\hline 5.Faringe & & & Constriç & & & & & & & \\
\hline & & & Expansấ & & & & & & & \\
\hline 6.Velofaringe & & & Escape & al audível & & & & & & \\
\hline & & & Nasal & & & & & & & \\
\hline & & & Denasal & & & & & & & \\
\hline 7. Altura de laringe & & & Elevada & & & & & & & \\
\hline & & & Abaixad & & & & & & & \\
\hline B. TENSÃO MUSCULAR & ERAL & & & & & & & & & \\
\hline 8. Tensão do trato vocal & & & Hiperfun & & & & $\mathrm{X}$ & & & \\
\hline & & & Hipofuns & & & & & & & \\
\hline 9. Tensão laríngea & & & Hiperfun & & & & $\mathrm{X}$ & & & \\
\hline & & & Hipofuns & & & & & & & \\
\hline C. ELEMENTOS FONATO & OS & & & & & & & & & \\
\hline & AJUSTE & & Present & & & aus & de e & cal & & \\
\hline & & & Neutro & Não & Mo & der & & & rem & \\
\hline & & & & Neutro & 1 & 2 & 3 & 4 & 5 & 6 \\
\hline 10. Modo de fonação & Modal & & & & & & & & & \\
\hline & Falsete & & & $X i$ & & & & & & \\
\hline & Crepitân & I vocal fry & & & & & & & & \\
\hline & Voz crep & & & & & & & & & \\
\hline 11. Fricção laríngea & Escape o & & & & & & & & & \\
\hline & Voz sopr & & & & & & & & & \\
\hline 12.Irreqularidade laríngea & Voz áspe & & & & & & & & & \\
\hline
\end{tabular}

Figura 4: Informante inexperiente A2. 


\begin{tabular}{|c|c|c|c|c|c|c|c|c|c|c|}
\hline \multirow{3}{*}{\begin{tabular}{|l} 
QUALIDADE VOCAL \\
\\
\end{tabular}} & \multicolumn{2}{|c|}{ PRIMEIRA PASSADA } & \multicolumn{8}{|c|}{ SEGUNDA PASSADA } \\
\hline & \multirow[t]{2}{*}{ Neutro } & \multirow{2}{*}{$\begin{array}{l}\text { Não } \\
\text { neutro }\end{array}$} & \multirow{2}{*}{\multicolumn{2}{|c|}{ AJUSTE }} & \multicolumn{3}{|c|}{ Moderado } & \multicolumn{3}{|c|}{ Extremo } \\
\hline & & & & & 1 & 2 & 3 & 4 & 5 & 6 \\
\hline \multicolumn{11}{|c|}{ A. ELEMENTOS DO TRATO VOCAL } \\
\hline \multirow{5}{*}{ 1.Lábios } & & & \multicolumn{2}{|c|}{ Arredondados/protraídos } & & & & & & \\
\hline & & & \multicolumn{2}{|c|}{ Estirados } & & & & & & \\
\hline & & & \multicolumn{2}{|c|}{ Labiodentalização } & & & & & & \\
\hline & & & \multicolumn{2}{|c|}{ Extensão diminuída } & & & & & & \\
\hline & & & \multicolumn{2}{|c|}{ Extensão aumentada } & & & & & & \\
\hline \multirow{5}{*}{ 2. Mandíbula } & & & \multicolumn{2}{|c|}{ Fechada } & & & & & & \\
\hline & & & \multicolumn{2}{|l|}{ Aberta } & & & & & & \\
\hline & & & \multicolumn{2}{|c|}{ Protraída } & & & & & & \\
\hline & & & \multicolumn{2}{|c|}{ Extensão diminuída } & & & & & & \\
\hline & & & \multicolumn{2}{|c|}{ Extensão aumentada } & & & & & & \\
\hline 3.Língua ponta/lâmina & & & Avançac & & & & & & & \\
\hline & & & Recuada & & & & & & & \\
\hline 4. Corpo de língua & & & Avançac & & & & & & & \\
\hline & & & Recuado & & & & & & & \\
\hline & & & Elevado & & & & & & & \\
\hline & & & Abaixad & & & & & & & \\
\hline & & & Extensã & minuída & & & & & & \\
\hline & & & Extensã & umentada & & & & & & \\
\hline 5.Faringe & & & Constriç & & & & & & & \\
\hline & & & Expansã & & & & & & & \\
\hline 6.Velofaringe & & & Escape & al audível & & & & & & \\
\hline & & & Nasal & & & & & & & \\
\hline & & & Denasal & & & & & & & \\
\hline 7. Altura de laringe & & & Elevada & & & & & & & \\
\hline & & & Abaixad & & & & & & & \\
\hline B. TENSÃO MUSCULAR & RAL & & & & & & & & & \\
\hline 8. Tensão do trato vocal & & & Hiperfun & & & & $\mathrm{X}$ & & & \\
\hline & & & Hipofuns & & & & & & & \\
\hline 9. Tensão laríngea & & & Hiperfun & & & & $\mathrm{X}$ & & & \\
\hline & & & Hipofuns & & & & & & & \\
\hline C. ELEMENTOS FONATÓ & OS & & & & & & & & & \\
\hline & AJUSTE & & Present & & Gr & aus & de & cal & & \\
\hline & & & Neutro & Não & Mo & der & & & rem & \\
\hline & & & & Neutro & 1 & 2 & 3 & 4 & 5 & 6 \\
\hline 10. Modo de fonação & Modal & & & $\mathrm{X}$ & & & & & & \\
\hline & Falsete & & & & & & & & & \\
\hline & Crepitânc & I vocal fry & & & & & & & & \\
\hline & Voz crep & & & & & $\mathrm{Xi}$ & & & & \\
\hline 11. Fricção laríngea & Escape d & & & & & & & & & \\
\hline & Voz sopr & & & & & $\mathrm{Xi}$ & & & & \\
\hline 12.Irregularidade laríngea & Voz áspe & & & $\mathrm{X}$ & & & & & & \\
\hline
\end{tabular}

Figura 5: Informante inexperiente I. 


\begin{tabular}{|c|c|c|c|c|c|c|c|c|c|c|}
\hline \multirow{3}{*}{\begin{tabular}{|l|} 
QUALIDADE VOCAL \\
\\
\end{tabular}} & \multicolumn{2}{|c|}{ PRIMEIRA PASSADA } & \multicolumn{8}{|c|}{ SEGUNDA PASSADA } \\
\hline & \multirow[t]{2}{*}{ Neutro } & \multirow{2}{*}{$\begin{array}{l}\text { Não } \\
\text { neutro }\end{array}$} & \multirow{2}{*}{\multicolumn{2}{|c|}{ AJUSTE }} & \multicolumn{3}{|c|}{ Moderado } & \multicolumn{3}{|c|}{ Extremo } \\
\hline & & & & & 1 & 2 & 3 & 4 & 5 & 6 \\
\hline \multicolumn{11}{|c|}{ A. ELEMENTOS DO TRATO VOCAL } \\
\hline \multirow{5}{*}{ 1.Lábios } & & & \multicolumn{2}{|c|}{ Arredondados/protraídos } & & & & & & \\
\hline & & & \multicolumn{2}{|c|}{ Estirados } & & & & & & \\
\hline & & & \multicolumn{2}{|c|}{ Labiodentalização } & & & & & & \\
\hline & & & \multicolumn{2}{|c|}{ Extensão diminuída } & & & & & & \\
\hline & & & \multicolumn{2}{|c|}{ Extensão aumentada } & & & & & & \\
\hline \multirow{5}{*}{ 2. Mandíbula } & & & \multicolumn{2}{|c|}{ Fechada } & & & & & & \\
\hline & & & \multicolumn{2}{|l|}{ Aberta } & & $\mathrm{X}$ & & & & \\
\hline & & & \multicolumn{2}{|l|}{ Protraída } & & & & & & \\
\hline & & & \multicolumn{2}{|c|}{ Extensão diminuída } & & & & & & \\
\hline & & & \multicolumn{2}{|c|}{ Extensão aumentada } & & & & & & \\
\hline 3.Língua ponta/lâmina & & & Avançad & & & & & & & \\
\hline & & & Recuada & & & & & & & \\
\hline 4. Corpo de língua & & & Avançad & & & & & & & \\
\hline & & & Recuado & & & & & & & \\
\hline & & & Elevado & & & & & & & \\
\hline & & & Abaixado & & & & & & & \\
\hline & & & Extensão & minuída & & & & & & \\
\hline & & & Extensão & umentada & & & & & & \\
\hline 5.Faringe & & & Constriçã & & & & & & & \\
\hline & & & Expansã & & & & & & & \\
\hline 6.Velofaringe & & & Escape $n$ & al audível & & & & & & \\
\hline & & & Nasal & & & & & & & \\
\hline & & & Denasal & & & & & & & \\
\hline 7. Altura de laringe & & & Elevada & & & & & $\mathrm{X}$ & & \\
\hline & & & Abaixada & & & & & & & \\
\hline B. TENSÃO MUSCULAR & ERAL & & & & & & & & & \\
\hline 8. Tensão do trato vocal & & & Hiperfun & & & & & & $\mathrm{x}$ & \\
\hline & & & Hipofunç & & & & & & & \\
\hline 9. Tensão laríngea & & & Hiperfun & & & & & & $\mathrm{x}$ & \\
\hline & & & Hipofunç & & & & & & & \\
\hline C. ELEMENTOS FONATC & OS & & & & & & & & & \\
\hline & AJUSTE & & Presente & & & aus & dee & cal & & \\
\hline & & & Neutro & & & der & & & tren & \\
\hline & & & & Neutro & 1 & 2 & 3 & 4 & 5 & 6 \\
\hline 10. Modo de fonação & Modal & & $\mathrm{X}$ & & & & & & & \\
\hline & Falsete & & & & & & & & & \\
\hline & Crepitân & I vocal fry & & & & & & & & \\
\hline & Voz crep & & & & & & & & & \\
\hline 11. Fricção laríngea & Escape c & & & & & & & & & \\
\hline & Voz sopr & & & & & & & & & \\
\hline 12.Irregularidade laríngea & Voz áspe & & & $\mathrm{Xi}$ & & & & & & \\
\hline
\end{tabular}

Figura 6: Informante experiente A. 


\begin{tabular}{|c|c|c|c|c|c|c|c|c|c|c|}
\hline \multirow{3}{*}{\begin{tabular}{|l} 
QUALIDADE VOCAL \\
\\
\end{tabular}} & \multicolumn{2}{|c|}{ PRIMEIRA PASSADA } & \multicolumn{8}{|c|}{ SEGUNDA PASSADA } \\
\hline & \multirow[t]{2}{*}{ Neutro } & \multirow{2}{*}{$\begin{array}{l}\text { Não } \\
\text { neutro }\end{array}$} & \multirow{2}{*}{\multicolumn{2}{|c|}{ AJUSTE }} & \multicolumn{3}{|c|}{ Moderado } & \multicolumn{3}{|c|}{ Extremo } \\
\hline & & & & & 1 & 2 & 3 & 4 & 5 & 6 \\
\hline \multicolumn{11}{|c|}{ A. ELEMENTOS DO TRATO VOCAL } \\
\hline \multirow{5}{*}{ 1.Lábios } & & & \multicolumn{2}{|c|}{ Arredondados/protraídos } & & & & & & \\
\hline & & & \multicolumn{2}{|l|}{ Estirados } & & & & & & \\
\hline & & & \multicolumn{2}{|c|}{ Labiodentalização } & & & & & & \\
\hline & & & \multicolumn{2}{|c|}{ Extensão diminuída } & & & & & & \\
\hline & & & \multicolumn{2}{|c|}{ Extensão aumentada } & & & & & & \\
\hline \multirow{5}{*}{ 2. Mandíbula } & & & \multicolumn{2}{|l|}{ Fechada } & & & & & & \\
\hline & & & \multicolumn{2}{|l|}{ Aberta } & & & & $\mathrm{X}$ & & \\
\hline & & & \multicolumn{2}{|l|}{ Protraída } & & & & & & \\
\hline & & & \multicolumn{2}{|c|}{ Extensão diminuída } & & $\mathrm{X}$ & & & & \\
\hline & & & \multicolumn{2}{|c|}{ Extensão aumentada } & & & & & & \\
\hline 3.Língua ponta/lâmina & & & Avançad & & & & & & & \\
\hline & & & Recuada & & & & & & & \\
\hline 4. Corpo de língua & & & Avançad & & & & & & & \\
\hline & & & Recuado & & & $\mathrm{X}$ & & & & \\
\hline & & & Elevado & & & & & & & \\
\hline & & & Abaixadc & & & & & & & \\
\hline & & & Extensão & ninuída & & $\mathrm{X}$ & & & & \\
\hline & & & Extensãc & Imentada & & & & & & \\
\hline 5.Faringe & & & Constriçấ & & & & & & & \\
\hline & & & Expansã & & & & & & & \\
\hline 6.Velofaringe & & & Escape $n$ & al audível & & & & & & \\
\hline & & & Nasal & & & & & & & \\
\hline & & & Denasal & & & & & & & \\
\hline 7. Altura de laringe & & & Elevada & & & & & & $\mathrm{x}$ & \\
\hline & & & Abaixada & & & & & & & \\
\hline B. TENSÃO MUSCULAR & RAL & & & & & & & & & \\
\hline 8. Tensão do trato vocal & & & Hiperfun & & & & & & & $\mathrm{x}$ \\
\hline & & & Hipofunç & & & & & & & \\
\hline 9. Tensão laríngea & & & Hiperfun & & & & & & & $\mathrm{x}$ \\
\hline & & & Hipofunç & & & & & & & \\
\hline C. ELEMENTOS FONATC & OS & & & & & & & & & \\
\hline & AJUSTE & & Presente & & & Ius & le e & cal & & \\
\hline & & & Neutro & Não & & der & & & tren & \\
\hline & & & & Neutro & 1 & 2 & 3 & 4 & 5 & 6 \\
\hline 10. Modo de fonação & Modal & & $\mathrm{X}$ & & & & & & & \\
\hline & Falsete & & & & & & & & & \\
\hline & Crepitânc & I vocal fry & & & & & & & & \\
\hline & Voz crep & & & & & & & & & \\
\hline 11. Friç̧ão laríngea & Escape o & & & & & & & & & \\
\hline & Voz sopr & & & & & & & & & \\
\hline 12.Irregularidade laríngea & Voz áspe & & & $\mathrm{Xi}$ & & & & & & \\
\hline
\end{tabular}

Figura 7: Informante experiente J. 